\title{
Boomerang latissimus dorsi flap in total breast reconstruction: report of three cases
}

\author{
Myeong Jae Kang ${ }^{1 \wedge}$, Tae Hyun Park ${ }^{1 \wedge}$, Jeong Yeop Ryu ${ }^{1 \wedge}$, Byeongju Kang ${ }^{2} \wedge$, Jeeyeon Lee ${ }^{2}$, \\ Ho Yong Park ${ }^{2}$, Jung Dug Yang ${ }^{1 \wedge}$, Joon Seok Lee ${ }^{1 \wedge}$ \\ ${ }^{1}$ Department of Plastic and Reconstructive Surgery, School of Medicine, Kyungpook National University, Daegu, Korea; ${ }^{2}$ Department of Surgery, \\ School of Medicine, Kyungpook National University, Daegu, Korea \\ Correspondence to: Joon Seok Lee, MD, PhD. Department of Plastic and Reconstructive Surgery, School of Medicine, Kyungpook National \\ University, Kyungpook National University Chilgok-Hospital, 807, Hoguk-ro, Buk-gu, Daegu 41404, Korea. Email: leejspo@knu.ac.kr.
}

\begin{abstract}
The latissimus dorsi (LD) flap is one of the most useful techniques for autologous breast reconstruction, especially in Asians. However, for reconstruction of moderate to large volume breasts, it is difficult to achieve the desirable volume and projection with the classic extended LD flap. Although there is the option of concurrent application of an LD flap and a small silicone implant, this may lead to larger volume than desired; furthermore, an increasing number of patients prefer reconstruction with autologous tissue alone. In this case report, we present satisfactory outcomes for moderate to large breast reconstruction in three patients, using the boomerang LD flap technique, a modification of the conventional extended LD flap. The flap was harvested with a transverse incision along the brassiere line and a vertical incision under the arm adjusted for the breast width of the patient. Then, after tunneling to the breast, the autologous reconstructed breast was created with the boomerang LD flap before moving the flap. There were no severe intraoperative or postoperative complications. In one case, linear necrosis of the breast wound was observed and the patient underwent revision operation for treatment. Seromas were not observed in the donor site of the boomerang LD flap and healing was successful. However, the donor site incision was 1.5 to 1.6 times longer than that of the classic LD flap. Boomerang LD flaps allow immediate breast reconstruction after neoadjuvant chemotherapy, without the need of microsurgery or use of abdominal-based flaps and without using silicone implants.
\end{abstract}

Keywords: Latissimus dorsi flap (LD flap); autologous breast reconstruction; large breast reconstruction

Submitted Aug 28, 2021. Accepted for publication Nov 17, 2021.

doi: 10.21037 /gs-21-595

View this article at: https://dx.doi.org/10.21037/gs-21-595

\section{Introduction}

The myocutaneous latissimus dorsi (LD) flap can be used for various cases of breast reconstructions. This flap can cover an extensive area with abundant blood supply from the thoracodorsal artery, and both the free flap and the pedicled flap are used for various reconstructions (1). After mastectomy for breast cancer, pedicled flaps are widely used for breast reconstruction according to breast volume and defect size.

In case of small breast reconstruction, filling the volume and projection to the desired level is possible with the use of extended LD flaps, but in case of moderate to large breasts, the use of extended LD flaps does not yield sufficient breast volume and projection, requiring alternative solutions. Recently, LD flaps have been used in combination with a

^ ORCID: Myeong Jae Kang, 0000-0002-3002-0614; Tae Hyun Park, 0000-0002-0373-9156; Jeong Yeop Ryu, 0000-0003-2812-5051; Byeongju Kang, 0000-0002-3589-5559; Jeeyeon Lee, 0000-0003-1826-1690; Ho Yong Park, 0000-0002-4380-0089; Jung Dug Yang, 00000002-9121-3604; Joon Seok Lee, 0000-0002-1580-0487. 

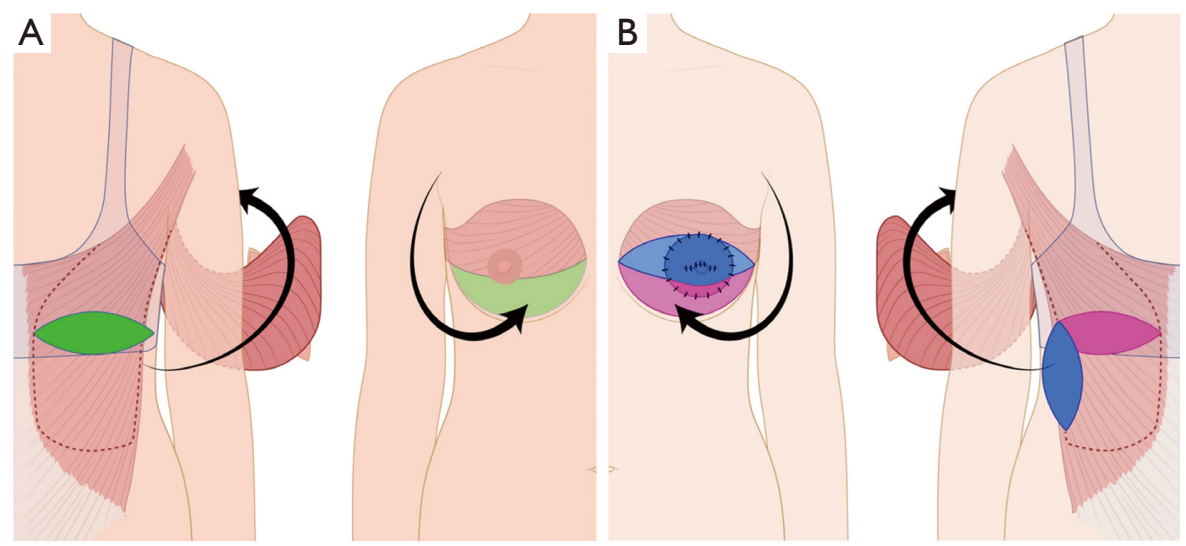

Figure 1 Illustration of total breast reconstruction using LD flap techniques. (A) Classic extended LD flap design. The long scar covered by the brassiere line is formed in the transverse direction to the ipsilateral back; (B) boomerang LD flap design. The design is implemented so that the transverse scar is formed along the mid-axillary line and hidden by the arm vertically in the upright position. The horizontal cutaneous flap is positioned in the upper pole area of the reconstructed breast, and the vertical cutaneous flap is positioned in the lower pole area. In the case of excision of nipple-areolar complex, simultaneous nipple reconstruction can also be performed. LD, latissimus dorsi.

small silicone implant for reconstruction, or fat injection is applied to the extended LD flap to increase the volume. Although the volume can be increased as desired by using the small silicone implant, it is difficult to accurately predict due to the atrophy of the LD flap, and there are possibilities of complications due to the implant, a foreign body. In addition, in the case of the method of using fat injection, multiple fat injection is generally required for the augmentation of the volume as desired, and there could be difficulties due to multiple procedures (2-4).

Furthermore, although gold standard reconstruction is possible with abdominal-based flaps [e.g., transverse rectus abdominis (TRAM) flap, deep inferior epigastric perforator (DIEP) flap, etc.], which use tissues from different sites, since the operation field is large, it is a long-time operation and the position of the umbilicus also has to be transferred properly, and it is a perforator flap among free flaps, not a concept of the pedicled flap, so micro-anastomosis is necessary. Moreover, in the case of injuries in the abdominal fascia or weakening, problems like herniation could occur at rare intervals. There are possibilities of these multiple complications.

In addition, if there is a previous history of abdominal surgery, this method is not the first choice. Therefore, in this report, we present three cases wherein a new Boomerang LD flap technique was applied with Boomerang shaped skin paddle modification of the classic LD flap design, to increase the overall volume of the reconstructed breast and improve the fullness of the upper pole as well as the projection, with concurrent primary closure of the donor site (Figure 1). We present the following cases in accordance with the CARE reporting checklist (available at https://gs.amegroups.com/ article/view/10.21037/gs-21-595/rc).

\section{Case presentation}

\section{Case 1}

The patient was a 40 -year-old woman with rightsided breast cancer of stage 3, human epidermal growth factor receptor 2 (HER2) (-), estrogen receptor (ER)/ progesterone receptor (PR) (-/-), with a tumor size of $4.5 \mathrm{~cm}$. Neoadjuvant chemotherapy was initiated in January 2021 and successfully completed. In June 2021, under general anesthesia, endoscopic skin sparing mastectomy, extending 4-5 $\mathrm{cm}$ to the lateral part of the inframammary fold (IMF), and sentinel lymph node biopsy (SLNB) were performed for the right-sided intraductal carcinoma (IDC). Thereafter, immediate breast reconstruction using a boomerang LD flap was performed. The dimensions of the boomerang LD flap were $6 \times 18$ and $5 \times 15 \mathrm{~cm}$. The resected breast tissue weighed $332 \mathrm{~g}$, whereas the boomerang LD flap weighed $320 \mathrm{~g}$. Three months postoperatively, the patient was satisfied with the outcome and had no major complications (Figure 2, Table 1).

\section{Case 2}

The patient was a 37-year-old woman with left-sided IDC. 

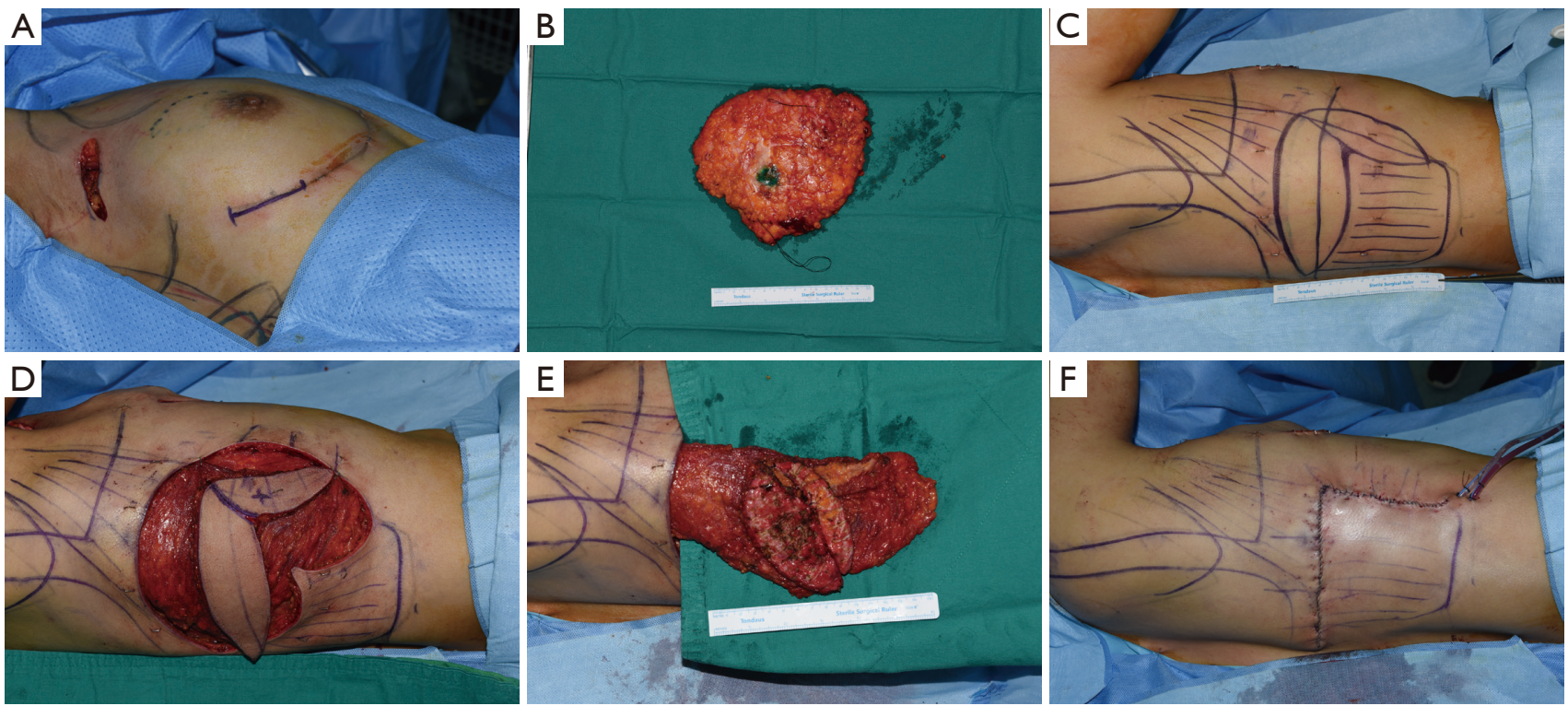

Figure 2 Case 1. Intraoperative findings of boomerang LD flap. (A) Endoscopic skin-sparing mastectomy with a 4-5 cm incision in the lateral part of the IMF; (B) excised mass after mastectomy; (C) boomerang LD flap design; (D) elevated boomerang LD flap. The X marks the perforator area checked by Doppler and used as the pivot point, (E) the transverse cutaneous flap and vertical cutaneous flap are formed into the reconstructed breast shape; (F) boomerang LD flap donor site, immediate postoperative view. LD, latissimus dorsi; IMF, inframammary fold.

The ER/PR/HER2 subtypes were HER2 (1+), ER/PR (-/-) and the tumor size was approximately $6.4 \mathrm{~cm}$. The patient underwent neoadjuvant chemotherapy from February to May 2021. In July 2021, under general anesthesia, left skin sparing mastectomy and SLNB were performed, combined with excision of the nipple-areolar complex (NAC). The mastectomy was immediately followed by breast reconstruction using boomerang LD flap (Figures 3,4). The dimensions of the boomerang LD flap were $8 \times 24$ and $6 \times$ $18 \mathrm{~cm}$, respectively. The resected breast tissue weighed $1,251 \mathrm{~g}$, whereas the boomerang LD flap weighed $1,065 \mathrm{~g}$ (Table 1). Simultaneous nipple reconstruction was also performed.

In the case of this patient, sono-guided evaluation was performed because severe postoperative flap swelling was observed, and it was identified that the blood flow of the pedicle is in good condition. It was identified as simple LD muscle swelling, not hematoma or active bleeding, seroma so compression dressing with the elastic band was not conducted, and about half of the suture stitch was temporarily removed to suppress the flap even for small tension and take a measure to not aggravate the venous congestion. As a result, the patient got better and improved from postoperative 5 days.

\section{Case 3}

The patient was a 48-year-old woman with left-sided IDC. The ER/PR/HER2 subtypes were HERs (-), ER/PR (-/-), and the tumor size was $2.4 \mathrm{~cm}$. The patient underwent neoadjuvant chemotherapy from January to June 2021. In July 2021, under general anesthesia, lateral incision was performed on the breast and skin sparing mastectomy and axillary lymph node dissection (ALND) were performed. This was followed by immediate breast reconstruction using boomerang LD flap. The dimensions of the boomerang LD flap were $7 \times 21$ and $6 \times 18 \mathrm{~cm}$, respectively. The resected breast tissue weighed $606 \mathrm{~g}$, whereas the boomerang LD flap weighed $470 \mathrm{~g}$ (Figure 5, Table 1).

All procedures performed in studies involving human participants were in accordance with the ethical standards of the institutional and/or national research committee(s) and with the Helsinki Declaration (as revised in 2013). Written informed was obtained from all patients for publication of this article and accompanying images. A copy of the written consent is available for review by the 
Table 1 Patient demographics

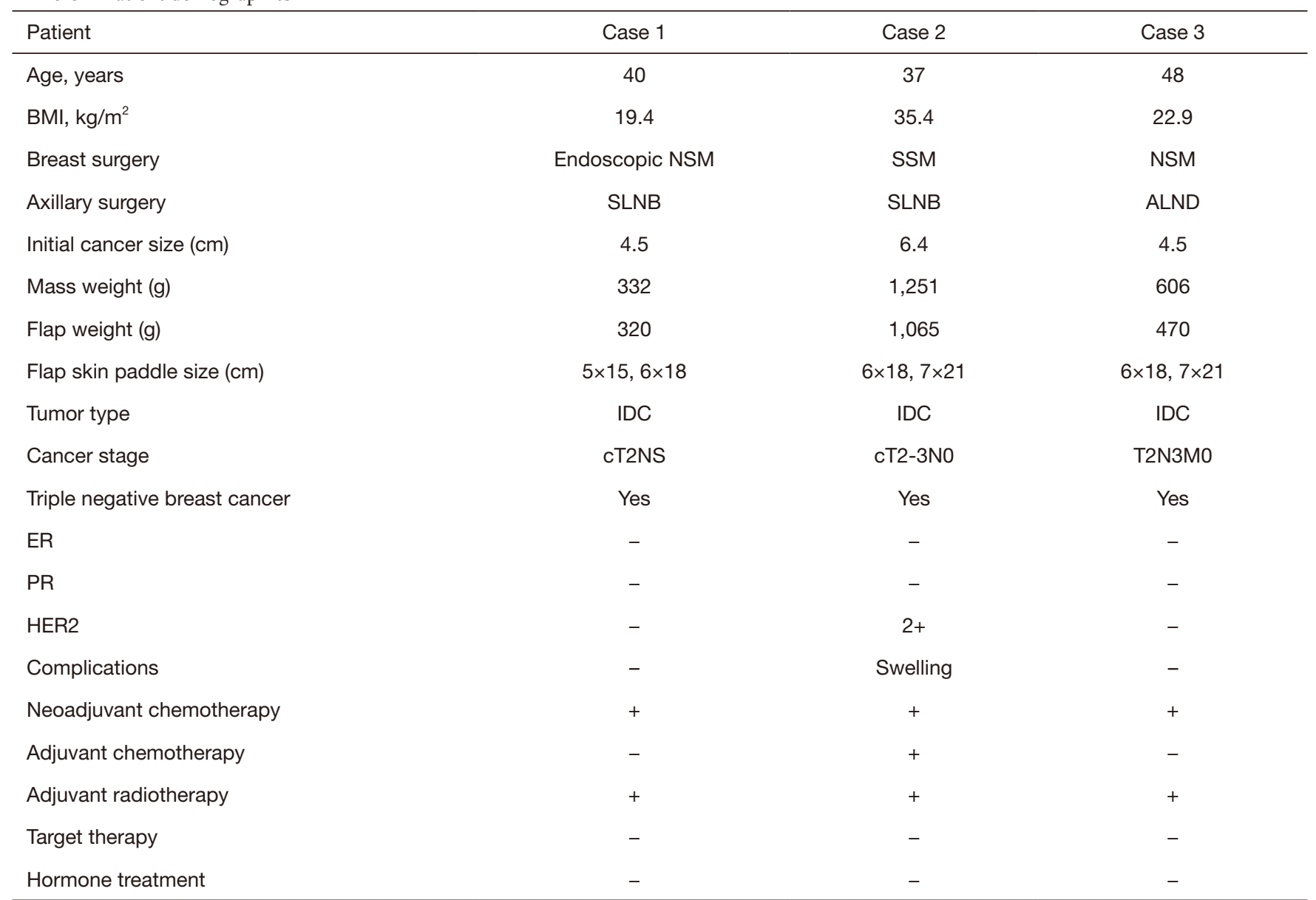

NSM, nipple sparing mastectomy; SSM, skin sparing mastectomy; SLNB, sentinel lymph node biopsy; ALND, axillary lymph node dissection; IDC, intraductal carcinoma; ER, estrogen receptor; PR, progesterone receptor.

editorial office of this journal.

\section{Boomerang LD flap: preoperative design and operative technique (Figure 1)}

On the side of the affected breast the brassiere line and the area covered by the arm in the upright position are marked. Considering the thickness of the skin flap, the long axis of the skin paddle of the horizontal LD flap is marked to match the patient's breast width, and the long axis of the vertical LD flap is marked to be about $1 \mathrm{~cm}$ smaller. The perforator is checked via Doppler around the hinge point where the two axes meet. As illustrated in Figure 1, this area is the part where the damage to the perforator is minimized when around $2 / 3$ of the skin paddle from the vertical LD flap is transposed to form the upper pole of the reconstructed breast. After marking the area, it must be ensured that the length of the widest part of the overlapping area of the horizontal and vertical skin paddles is less than $8 \mathrm{~cm}$ in consideration of the primary closure of the donor site. Following the marking of the area, the boomerang LD flap is lifted by inserting an incision, and for the vertical LD flap, which is responsible for the fullness of the upper pole of the reconstructed breast, the autologous breast is formed into the skin paddle of the boomerang shape by undermining the subcutaneous fat while paying attention to the perforator. Further, de-epithelization of the unnecessary epidermis is performed. If skin is required, it can be filled by approximation according to the size of the excised area, or in the case of NAC excision, simultaneous nipple reconstruction is also possible. Thereafter, the breast 

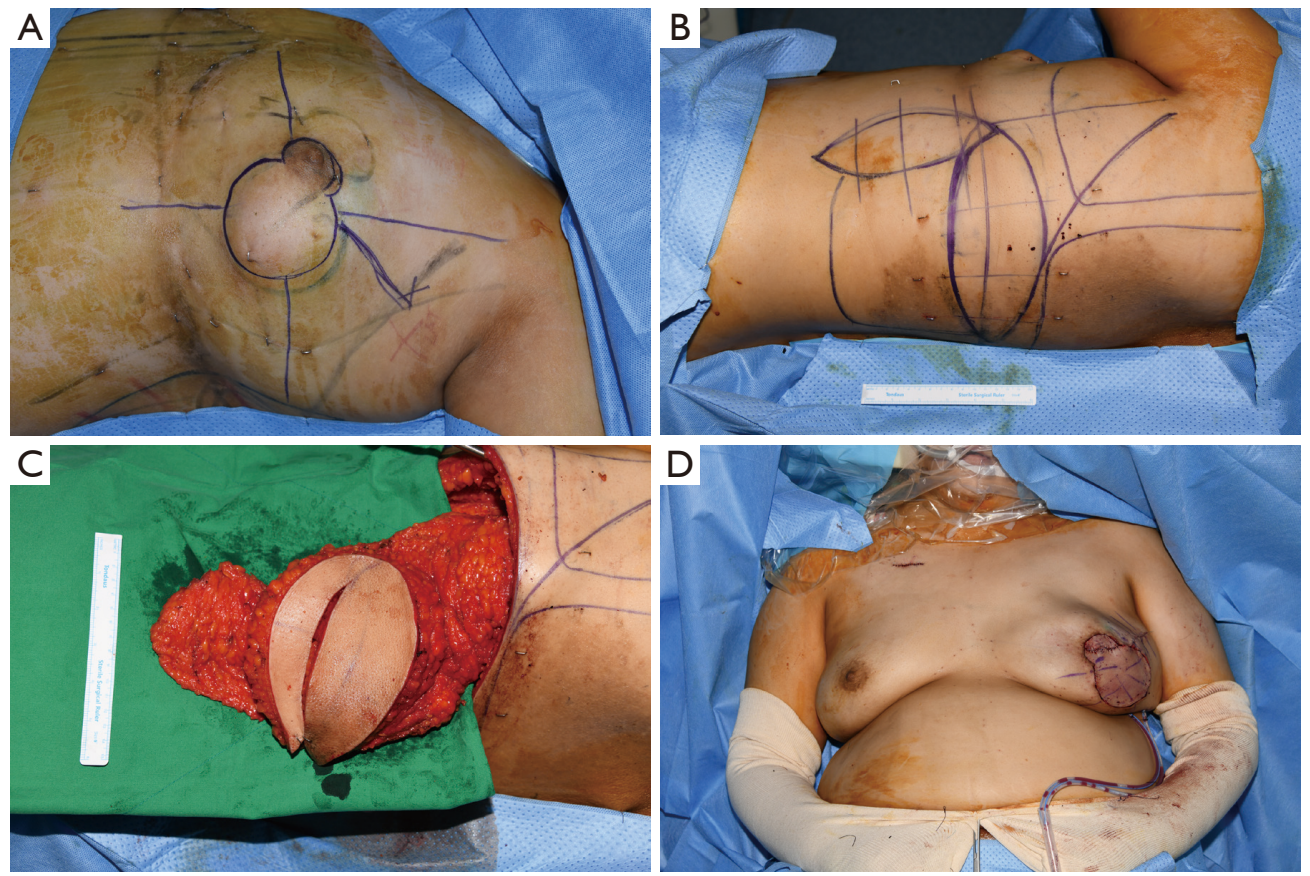

Figure 3 Case 2. Intraoperative findings of boomerang LD flap. (A) Excision design extending from nipple-areolar complex to part of breast skin; (B) boomerang LD flap design; (C) the transverse cutaneous flap and vertical cutaneous flap are formed into the reconstructed breast shape. The flap weight was over $1 \mathrm{~kg}$; (D) immediate postoperative findings with simultaneous nipple reconstruction also performed. LD, latissimus dorsi.
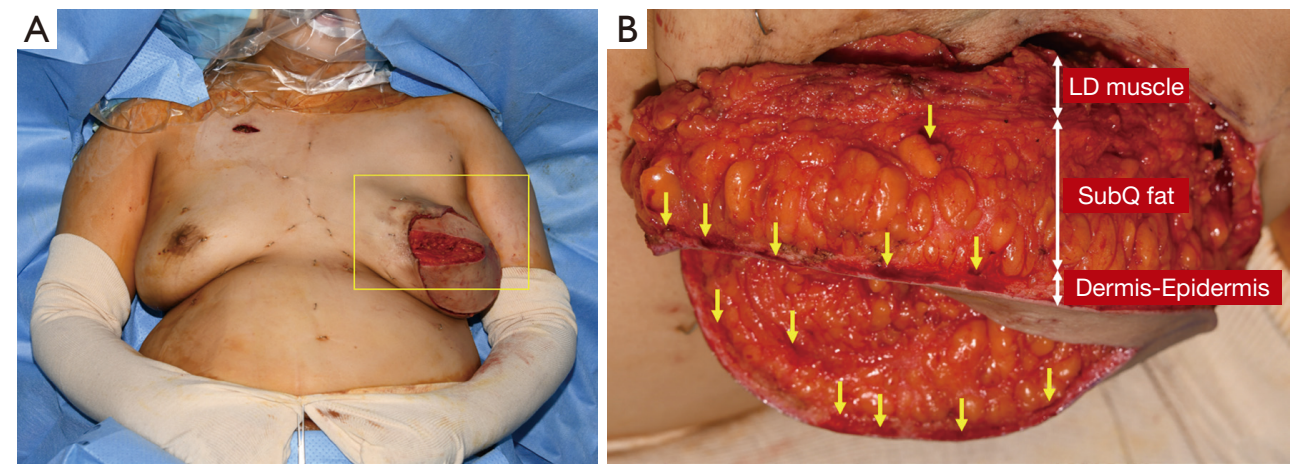

Figure 4 Confirmation of flap conditions. (A) Boomerang LD flap was transferred to the breast site; (B) pinpoint bleeding (yellow arrow) was observed in the dermis area of each skin flap. Subcutaneous fat accounts for a large portion of the reconstructed breast volume in both the transverse flap and the vertical flap. LD, latissimus dorsi.

shape is constructed using 2-0 Vicryl and the boomerang LD flap is transferred to the defect site of the breast through tunneling in the axillary area. Subsequently, $800 \mathrm{cc}$ suction drain is placed at the donor site in two lines and primary closure of the donor site is performed. At this time, closure is performed with approximation to maximize the distribution of the tension. Next, the patient is rolled to a supine position and the LD muscle in the most distal part of the boomerang LD flap is fixed to the uppermost pectoralis muscle of the reconstructed breast in 3-5 points using 2-0 Vicryl. The contour around the IMF is checked and suture is not performed in this area to minimize the possibility of postoperative deformity. When the boomerang LD flap is inset into breast shape, 

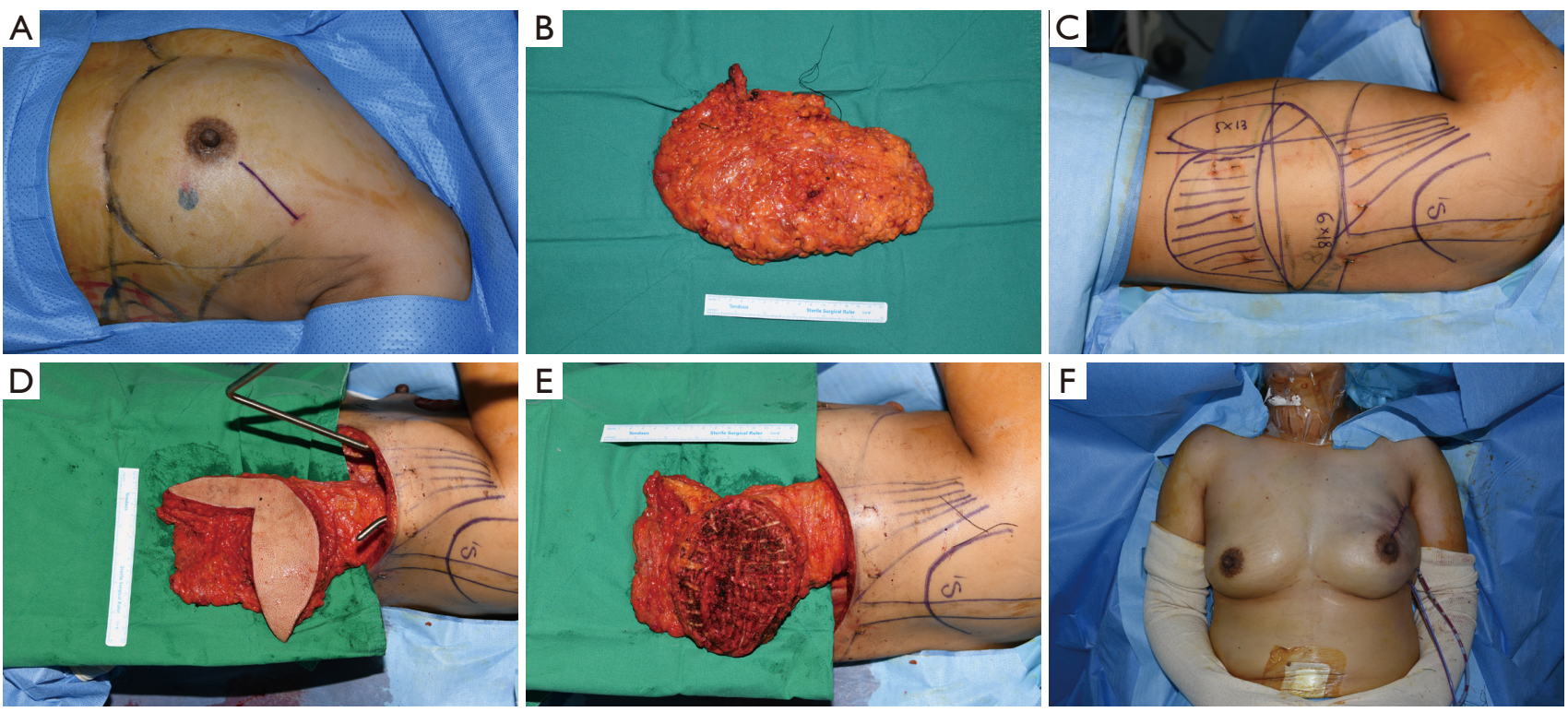

Figure 5 Case 3. Intraoperative findings of boomerang LD flap. (A) Single incision for skin-sparing mastectomy; (B) excised mass after mastectomy; (C) boomerang LD flap design; (D) elevated boomerang LD flap; (E) the transverse cutaneous flap and vertical cutaneous flap are formed into the reconstructed breast shape. The yellow line moves to the IMF of the reconstructed breast, and the LD distal muscle part, whereas the white line area is transposed to the upper pole. If the breast skin is not excised, de-epithelization is performed; (F) donor site, immediate postoperative findings. LD, latissimus dorsi.

400 cc suction drain is placed in the upper and lower sides of the flap, and then the primary closure is performed to complete the surgery.

\section{Discussion}

The LD flap is very useful for reconstruction of various parts of the body such as when coverage of a large defect due to trauma is required, when autologous breast reconstruction is required after mastectomy for treatment of breast cancer, and when coverage is required for a large defect after wide excision of malignant sarcoma. The use of LD flap was first reported by Iginio Tansini in 1960 and was first used for breast reconstruction in the 1970s (5-7). It is one of the very useful techniques for breast reconstruction for small to moderate breast volume as is often the case with Asians. In this case, with pedicled flap as the base, the flap is transposed by axillary tunneling. Since this is not a free flap such as abdominalbased flaps (TRAM and DIEP) or gluteus flaps, but a pedicled flap, it poses less burden for surgeons and causes no complications from anastomosis for patients, thus facilitating recovery. The use of pedicled TRAM flap is also possible, but there is a significant difference in the area of the operation field, and the operation time is also considerably longer. In addition, it has been reported that immediate breast reconstruction after mastectomy is safe and has cosmetic and psychological benefits (8-12) with minimal postoperative complications. Other studies have reported that breast reconstruction after mastectomy with autologous tissue or implants does not increase the recurrence rate of breast cancer $(11,12)$.

Despite this information patients who have undergone neoadjuvant chemotherapy and are surgical candidates sometimes have a different perspective as the mental distress and psychological toll that accompany a cancer diagnosis are profound (13-16). They strongly desire no recurrence of the breast cancer and no exposure to risk for other malignancies such as breast implant associated anaplastic large cell lymphoma or other side effects linked to the use of silicone which, in effect, is a foreign body (17-20), including the need for revision operation due to complications such as capsular contracture and rupture. For these reasons, most patients are strongly against the idea of reconstruction using prosthesis and most of them choose a surgical method with less risk of postoperative complications rather than a method resulting in shorter scar length. In patients with small breast volume, reconstruction can be performed 


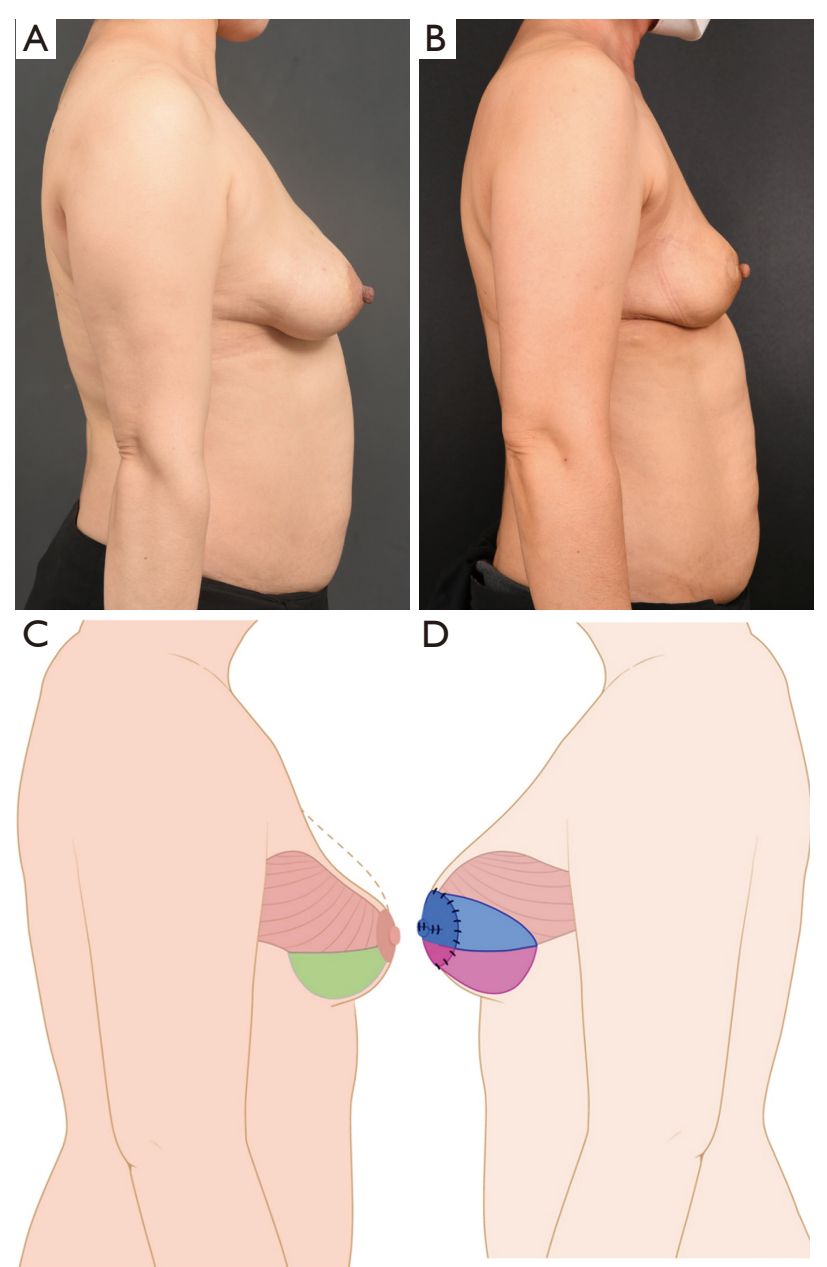

Figure 6 Case examples of insufficient breast volume in breast reconstruction using extended LD flap. (A) Preoperative photo; (B) 1 year postoperatively. It can be seen that the projection of the reconstructed breast is low, and the fullness of the upper pole is undesirable. Illustrations of lateral views in extended LD flap (C) and boomerang LD flap (D). In Asian women with a small to moderate breast volume, breast reconstruction using the classical extended LD flap is possible as shown in $(\mathrm{B}, \mathrm{C})$. However, when reconstruction of moderate to large breast volume is required, it is difficult to reconstruct the fullness of the upper pole with the LD muscle alone. To address this problem, a boomerang-shaped flap design can be employed with satisfactory outcomes. LD, latissimus dorsi.

using the classic extended LD flap, but for patients with moderate to large-sized breasts, who refuse abdominalbased flaps or have a contraindication for them, there are not many methods available for breast reconstruction using autologous tissue. It is possible to use fat grafting, but the survival rate of the fat cells may be different than expected causing difficulties in practice. Therefore, in this report, a boomerang-shaped modified LD flap design was proposed for improvement of the reconstructed breast volume, especially in the upper pole area, and the projection of the reconstructed breast (Figure 6). In planning the procedure, we found useful information in a paper published in 2013 by Zhang et al., which reported breast reconstruction with multilobed design (21). In essence, the idea of using a flap with abundant blood supply from the thoracodorsal artery is the same but in terms of designing the skin paddle, where the largest volume can be achieved, the proposed flap design enables increase of the volume by about $1.5-1.8$ times that of the classical extended LD flap. In the design of vertical LD flap to be placed in the upper pole area of the reconstructed breast, $2 / 3$ of the skin flap needs to be repositioned at subcutaneous level. To check and monitor the blood supply, the intraoperative pinpoint bleeding in the distal part of the flap is marked and continuously monitored during surgery (Figure 4). In addition, although the postoperative scar is long, with a $90^{\circ}$ angle between the horizontal and vertical scar, the increase in the length is less than twice that of the other methods. Furthermore, the scar is in the mid-axillary line and can be covered or hidden in the upright position, thereby representing an acceptable esthetic option (Figures 2,3,5,7,8). An additional important point of consideration is that, since performing primary closure of defects larger than $8 \mathrm{~cm}$ is difficult, the design should be implemented so that the length of diagonal line of the bent part of the boomerang flap, which is the widest part, should not exceed $8 \mathrm{~cm}$. The flap designs previously reported were kite-shaped or wing-shaped $(22,23)$, but with these designs it is difficult to hide the scar and the back skin contour may also appear irregular. On the contrary, with our modified LD flap, the vertical scar can be covered or hidden by the brassiere line and the arms. There were no major complications in the donor site 3 months or more after surgery, and although generalizations cannot be made because there are only three cases, the healing of the back donor site was faster than in cases of conventional extended LD flaps. In terms of patient satisfaction, all three patients were satisfied with the outcomes in terms of shape and volume of the breast and the lack of complications. Systematic surveys are conducted to include more patient groups in the future.

In conclusion, the boomerang LD flap is considered a useful autologous flap that can be applied for breast 

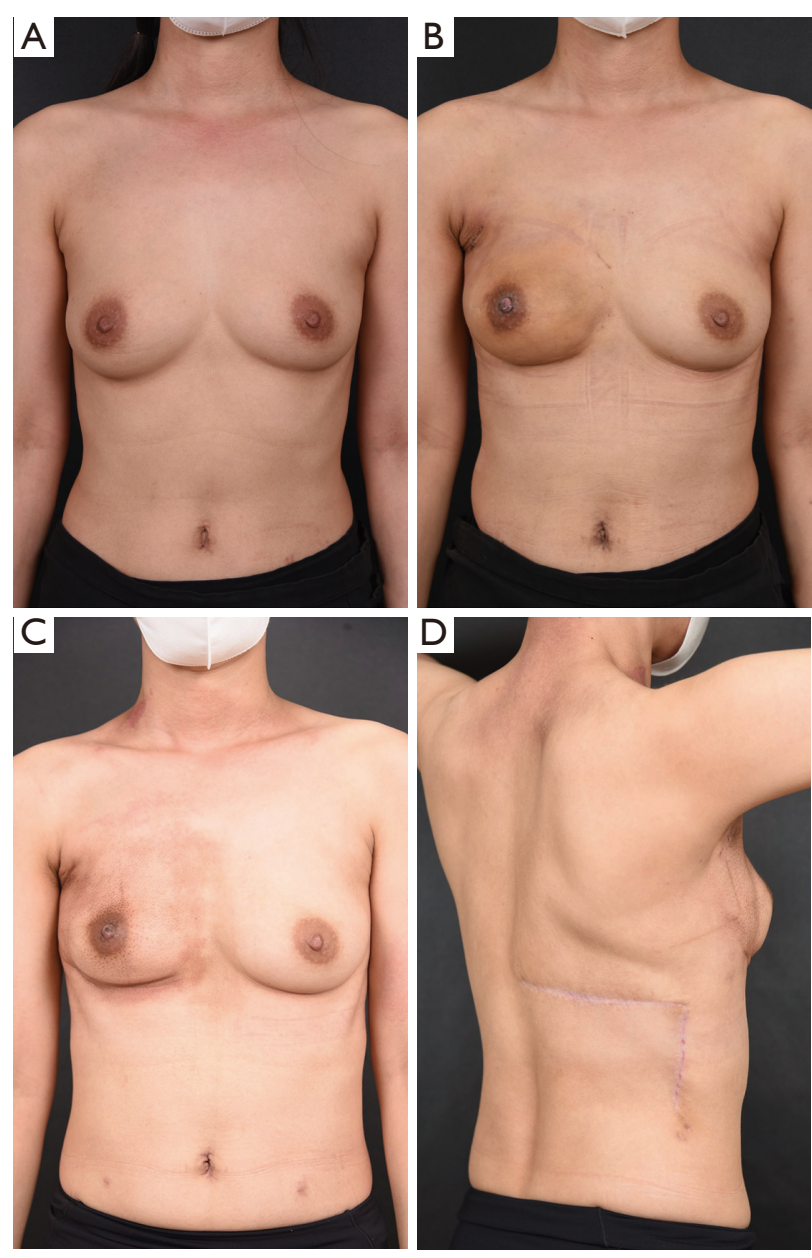

Figure 7 Case 1. (A) Preoperative photo; (B) 1 month postoperatively; (C,D) 5 months postoperatively. Anteroposterior view and donor site of boomerang $\mathrm{LD}$ flap. LD, latissimus dorsi.

reconstruction in patients with large breast volume who want to avoid implants. Satisfactory outcomes were achieved for the reconstructed breast, primary closure was possible at the donor site and the length of recovery period was similar to that of breast reconstruction using classic LD flaps.

\section{Acknowledgments}

Funding: None.

\section{Footnote}

Reporting Checklist: The authors have completed the CARE reporting checklist. Available at https://gs.amegroups.com/
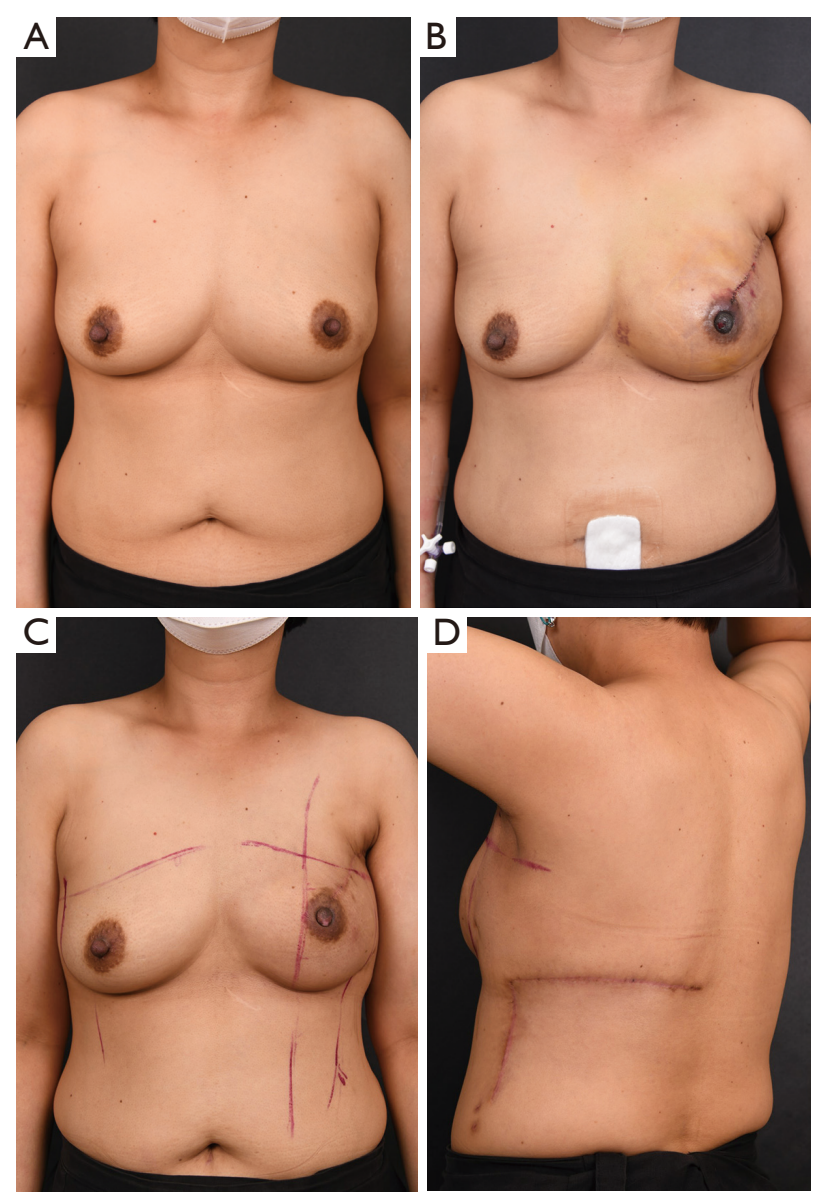

Figure 8 Case 3. (A) Preoperative photo; (B) 1 week postoperatively; (C,D) 4 months postoperatively. Anteroposterior view and donor site of boomerang LD flap. LD, latissimus dorsi.

article/view/10.21037/gs-21-595/rc

Peer Review File: Available at https://gs.amegroups.com/ article/view/10.21037/gs-21-595/prf

Conflicts of Interest: All authors have completed the ICMJE uniform disclosure form (available at https://gs.amegroups. com/article/view/10.21037/gs-21-595/coif). The authors have no conflicts of interest to declare.

Ethical Statement: The authors are accountable for all aspects of the work in ensuring that questions related to the accuracy or integrity of any part of the work are appropriately investigated and resolved. All procedures performed in human participants were in accordance with the ethical standards of the institutional and/or national 
research committee(s) and with the Helsinki Declaration (as revised in 2013). Informed consent for publication was obtained from all three patients. A copy of the written consent is available for review by the editorial office of this journal.

Open Access Statement: This is an Open Access article distributed in accordance with the Creative Commons Attribution-NonCommercial-NoDerivs 4.0 International License (CC BY-NC-ND 4.0), which permits the noncommercial replication and distribution of the article with the strict proviso that no changes or edits are made and the original work is properly cited (including links to both the formal publication through the relevant DOI and the license). See: https://creativecommons.org/licenses/by-nc$\mathrm{nd} / 4.0 /$.

\section{References}

1. Sood R, Easow JM, Konopka G, et al. Latissimus Dorsi Flap in Breast Reconstruction: Recent Innovations in the Workhorse Flap. Cancer Control 2018;25:1073274817744638.

2. Santanelli di Pompeo F, Laporta R, Sorotos M, et al. Latissimus dorsi flap for total autologous immediate breast reconstruction without implants. Plast Reconstr Surg 2014;134:871e-9e.

3. Economides JM, Song DH. Latissimus Dorsi and Immediate Fat Transfer (LIFT) for Complete Autologous Breast Reconstruction. Plast Reconstr Surg Glob Open 2018;6:e1656.

4. Demiri EC, Dionyssiou DD, Tsimponis A, et al. Outcomes of Fat-Augmented Latissimus Dorsi (FALD) Flap Versus Implant-Based Latissimus Dorsi Flap for Delayed Postradiation Breast Reconstruction. Aesthetic Plast Surg 2018;42:692-701.

5. Ribuffo D, Cigna E, Gerald GL, et al. Iginio Tansini revisited. Eur Rev Med Pharmacol Sci 2015;19:2477-81.

6. Olivari N. The latissimus flap. Br J Plast Surg 1976;29:126-8.

7. Mühlbauer W, Olbrisch R. The latissimus dorsi myocutaneous flap for breast reconstruction. Chir Plastica 1977;4:27-34.

8. Schaverien MV, Munnoch DA. Effect of neoadjuvant chemotherapy on outcomes of immediate free autologous breast reconstruction. Eur J Surg Oncol 2013;39:430-6.

9. Spiegel AJ, Butler CE. Recurrence following treatment of ductal carcinoma in situ with skin-sparing mastectomy and immediate breast reconstruction. Plast Reconstr Surg 2003;111:706-11.

10. Mustonen P, Lepistö J, Papp A, et al. The surgical and oncological safety of immediate breast reconstruction. Eur J Surg Oncol 2004;30:817-23.

11. Lee SB, Lee JW, Kim HJ, et al. Long-term outcomes of patients with breast cancer after nipple-sparing mastectomy/skin-sparing mastectomy followed by immediate transverse rectus abdominis musculocutaneous flap reconstruction: Comparison with conventional mastectomy in a single center study. Medicine (Baltimore) 2018;97:e0680.

12. Adam H, Bygdeson M, de Boniface J. The oncological safety of nipple-sparing mastectomy - a Swedish matched cohort study. Eur J Surg Oncol 2014;40:1209-15.

13. Santosa KB, Qi J, Kim HM, et al. Long-term PatientReported Outcomes in Postmastectomy Breast Reconstruction. JAMA Surg 2018;153:891-9.

14. Eltahir Y, Krabbe-Timmerman IS, Sadok N, et al. Outcome of Quality of Life for Women Undergoing Autologous versus Alloplastic Breast Reconstruction following Mastectomy: A Systematic Review and MetaAnalysis. Plast Reconstr Surg 2020;145:1109-23.

15. Toyserkani NM, Jørgensen MG, Tabatabaeifar S, et al. Autologous versus implant-based breast reconstruction: A systematic review and meta-analysis of Breast-Q patient-reported outcomes. J Plast Reconstr Aesthet Surg 2020;73:278-85.

16. Wu ZY, Han HH, Kim HJ, et al. A propensity scorematched comparison of recurrence outcomes after immediate implant vs autologous flap reconstruction in patients receiving neoadjuvant chemotherapy for breast cancer. Breast Cancer Res Treat 2021;187:417-25.

17. Santanelli di Pompeo F, Sorotos M. EURAPS Editorial: BIA-ALCL, a brief overview. J Plast Reconstr Aesthet Surg 2018;71:785-7.

18. Kim B, Roth C, Chung KC, et al. Anaplastic large cell lymphoma and breast implants: a systematic review. Plast Reconstr Surg 2011;127:2141-50.

19. Leberfinger AN, Behar BJ, Williams NC, et al. Breast Implant-Associated Anaplastic Large Cell Lymphoma: A Systematic Review. JAMA Surg 2017;152:1161-8.

20. Collett DJ, Rakhorst H, Lennox P, et al. Current Risk Estimate of Breast Implant-Associated Anaplastic Large Cell Lymphoma in Textured Breast Implants. Plast Reconstr Surg 2019;143:30S-40S.

21. Zhang YX, Messmer C, Pang FK, et al. A novel design of the multilobed latissimus dorsi myocutaneous flap to 
achieve primary donor-site closure in the reconstruction of large defects. Plast Reconstr Surg 2013;131:752e-8e.

22. Saha S, Ranjan P, Dhar A, et al. Wing-Shaped Skin Component of Latissimus Dorsi Myocutaneous Flap Design for Breast Reconstruction-Useful Innovation. Indian J Surg 2021;83: 1426-32 .

Cite this article as: Kang MJ, Park TH, Ryu JY, Kang B, Lee J, Park HY, Yang JD, Lee JS. Boomerang latissimus dorsi flap in total breast reconstruction: report of three cases. Gland Surg 2022;11(1):290-299. doi: 10.21037/gs-21-595
23. Casella D, Nanni J, Lo Torto F, et al. Extended Latissimus Dorsi Kite Flap (ELD-K Flap): Revisiting an Old Place for a Total Autologous Breast Reconstruction in Patients with Medium to Large Breasts. Aesthetic Plast Surg 2021;45:390-401. 